\title{
The bimodal metallicity distribution function of the globular clusters in the Galaxy: halo disc complementarity
}

\author{
E. Casuso ${ }^{1}$ and J. E. Beckman ${ }^{1,2}$ \\ 1 Instituto de Astrofísica de Canarias, 38200 La Laguna, Tenerife, Spain \\ e-mail: eca@ll.iac.es \\ 2 Consejo Superior de Investigaciones Científicas, Spain \\ e-mail: jeb@ll.iac.es
}

Received 7 August 2005 / Accepted 31 October 2005

\section{ABSTRACT}

\begin{abstract}
Aims. Our aim in this paper is to present an explanatory scenario for the formation of the observed relatively metal rich globular clusters associated with the thick disc of the Galaxy, distinct from the mode of formation of the lower metallicity halo clusters.

Methods. The observations to be accounted for here are the two peaks in the metallicity distribution of the thick disc globular clusters, at $[\mathrm{Fe} / \mathrm{H}] \sim-0.7$ and at $[\mathrm{Fe} / \mathrm{H}] \sim-0.4$. The first step is to verify the statistical significance of these peaks, and the insignificance of a much smaller peak at $[\mathrm{Fe} / \mathrm{H}] \sim-0.2$. The basic model assumption is that these globular clusters were formed as the most massive long term survivors of a much larger set of open clusters whose epochs of formation coincided with the main epochs of star formation in the thin disc. These latter are identified using established data sets giving the local stellar frequency distribution in time based on stellar activity indices.

Results. Our simple stellar accretion model accounts reasonably for the presence of the observed peaks in the cluster metallicity distribution, and the long time constant for the accretion as a massive cluster moves through the stellar environment explains qualitatively why the most recent peak in the local star formation rate has not yet given rise to a corresponding peak in the globular cluster distribution. It also explains in broad terms how a uniform process of cluster formation originating both open clusters and disc globular clusters can yield the observed high numbers of open clusters and the few surviving globulars.
\end{abstract}

Key words. Galaxy: abundances - Galaxy: globular clusters: general - Galaxy: kinematics and dynamics

\section{Introduction}

Globular clusters contain only a small fraction of the total stellar mass of galaxies, but provide tracers which can be used to shed significant light on models of galaxy formation and evolution. The globular clusters (GCs) of the Milky Way define geometrically a spheroidal spatial structure which has traditionally be called the "old halo" of the Galaxy, and extends out to some $40 \mathrm{kpc}$ from the Galactic centre. At distances beyond $40 \mathrm{kpc}$ the few observed clusters in the outer halo join with several of the dwarf galaxy satellites of the Milky Way to form a much larger scale planar distribution which almost certainly has a distinct origin and a different history (Harris 1976; Zinn 1985; Majewski 1994). The total number of known globular clusters in the Galaxy is close to 150 (Harris 1996). The presence of two major and remarkably distinct sub-populations of GCs, based on criteria of both metallicity and kinematics was suspected long ago (Kinman 1959), and was established clearly by Zinn (1985). The metal poor component contains around three quarters of the clusters, and is spread throughout the halo, while the metal rich component contains the remaining one quarter of the clusters and is almost entirely restricted to within the Solar circle, at galactocentric distances of less than $8 \mathrm{kpc}$. The complete metallicity distribution function (MDF), say in $[\mathrm{Fe} / \mathrm{H}]$, can be quite well described by a combination of two Gaussians, with a clear separation of rather more than 1 dex in metallicity between their peaks, which suggests distinct evolutionary histories for the two groups. Zinn (1985) and Armandroff (1989) interpreted the metal rich clusters as a disc population not only because of their high metallicities, but also because of their large systemic rotation velocity.

A model, which has become popular recently, to explain the bimodality in the observed MDF of the globular clusters is that of interactions of minor galaxies with the Milky Way (Bekki \& Chiba 2002; Li et al. 2004). The suggestion is that tidal interactions between galaxies dramatically change the formation rates of both field stars and globular clusters due to tidal compression of gas clouds, and their relatively efficient conversion into stars (Noguchi \& Ishibashi 1986; Ashman \& Zepf 1992; Kennicutt 1998; Bekki \& Couch 2001; Bekki et al. 2002). A recent and very interesting application of this mechanism is by Bekki et al. (2004) in which the authors explain the observed age gap of 
globular clusters in the Large Magellanic Cloud (LMC) by following the evolution of the LMC as it orbits the Galaxy and interacts with the Small Magellanic Cloud (SMC). However, the MDF of Galactic globular clusters requires a rather different explanation. The reason for this can be seen in Fig. 1 of Bekki et al. (2004). While the SMC and the LMC have gradually approached one another during their evolution, the effective mean distances between the SMC or the LMC and the Milky Way have changed relatively slowly during the lifetime of the Galactic disc, which implies that this kind of interaction cannot cope so well with the long term trends in the observational data. However other interactions of this kind cannot be ruled out as triggers of star and cluster formation. The thick disk, for example has been explained as the sequel of an early merger with a satellite galaxy (see e.g. Robin et al. 1996) and we know that the Sag dwarf galaxy is in the process of being incorporated into the Milky Way, with the interactive processes implied. However for the chemical evolution parameters to be satisfactorily explained, such interactions would have to be accompanied by the inflow of low metallicity gas.

Vandenberg (2000) derived the ages of 26 Galactic globular clusters with values of $[\mathrm{Fe} / \mathrm{H}]$ between -2.5 and -0.5 (Fig. 40 of the cited article) obtaining a global decrease in age from $\sim 14$ Gyr for the low metallicity population to around $\sim 8 \mathrm{Gyr}$ for that with higher metallicity. Although the absolute ages are subject to fairly large errors, these could not account for this major difference. Furthermore the difference is comparable to that inferred for the populations of halo and disc field stars (Vandenberg 2000) which points to a possible causal relation linking star formation in the disc in general with the formation of GCs in the disc, in the thick disc, and in the halo.

Our model is designed to explain the properties of the metal rich clusters including their kinematics. We do not try to determine the properties of the metal poor clusters with thick disc kinematics (Dinescu et al. 1999) which must have formed at a distinct epoch and under distinct conditions, and require a further modelling exercise which we have not attempted here. We note here that there is a debate in the literature about whether the metal rich clusters should be identified with the thick disc or the bulge. Specifically, the metal rich globular clusters within $4 \mathrm{kpc}$ of the Galactic centre are kinematically correlated with the HI in the bulge/bar (Minniti 1996; Cote 1999). However Heasley et al. (2000) found that the close similarity of the ages and metallicities of two bulge globular clusters (NGC 6624 and NGC 6637) to those of the thick disc globular clusters 47 Tuc and NGC 6342 indicates that the age-metallicity relations of these populations intersect, pointing to the possibility that these have a common origin. In Sect. 2 of this article we present the model, in Sect. 3 we compare our theoretical results with the observations, and in Sect. 4 we derive and discuss our conclusions.

\section{A model to account for the observed MDFs}

If one compares the MDF of the stars in the solar neighbourhood to that of the Galactic globular clusters, as presented in Fig. 1, one finds an interesting relation between their peaks. There are two principal peaks in both distributions, which

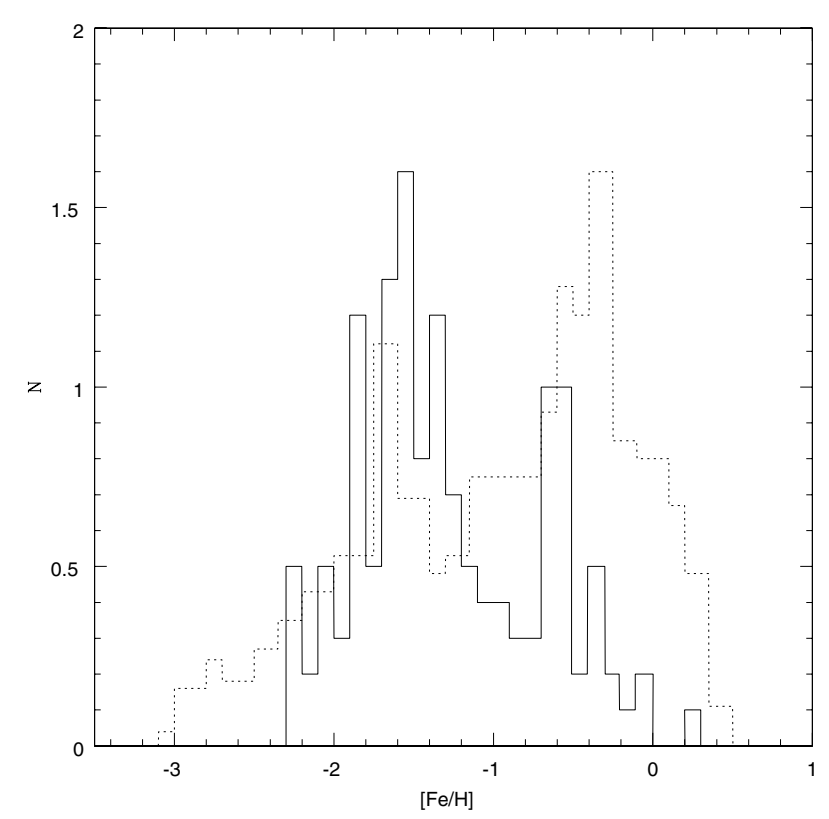

Fig. 1. Metallicity distribution functions for comparison: full line are the data corresponding to the globular clusters, taken from Harris (1999); dashed line are the data corresponding to the stars in the solar neighbourhood, taken from Carney et al. (1990). The bin taken for $[\mathrm{Fe} / \mathrm{H}]$ is $0.1 \mathrm{dex}$. Is there any complementarity?

correlate well in value of metallicity, but anticorrelate in frequency. This suggests some kind of complementarity in the events leading to the two distributions. In this context Jehin et al. (1998), using high resolution spectra, established correlations between the relative abundances of 16 elements in field halo stars and globular clusters, finding two populations which differ in the variation of the relative abundances of the s-process elements, on the one hand, and the r-process and alpha elements on the other. Their inference was that there exists a relation between the origin of the field halo stars and the globular clusters in the halo.

If we examine Fig. 1 we can see that in the metal rich peak of the globular cluster MDF there are two secondary peaks, which are outside the error bars of the observations (see Harris 1999, and also the results of our KMM test, see below). There is a highly significant peak at $[\mathrm{Fe} / \mathrm{H}]=-0.6$, and a smaller peak at $[\mathrm{Fe} / \mathrm{H}]=-0.35$. We have used the KMM algorithm (Ashman et al. 1994) to study the question of the bimodality of the metal rich galactic globular cluster MDF data of Harris (1999). This study yielded the following results. The p-value (the probability that the likelihood ratio test statistic would be at least as large as the observed value if the null hypothesis were true, i.e. the probability of determining the observed values from a sample distribution drawn from a unimodal Gaussian) for the data set is 0.01 , which indicates a significant rejection of the unimodal hypothesis. The algorithm in fact assigns 29 clusters to a set of data centred at a mean value of $[\mathrm{Fe} / \mathrm{H}]$ of -0.65 , and 16 clusters to another set centred at a mean $[\mathrm{Fe} / \mathrm{H}]$ of -0.30 with a common variance of 0.015 and a confidence level of $97 \%$. This result implies that the two small peaks close to $[\mathrm{Fe} / \mathrm{H}]=0.0$ are not statistically significant, but the two main peaks are statistically robust. We used 


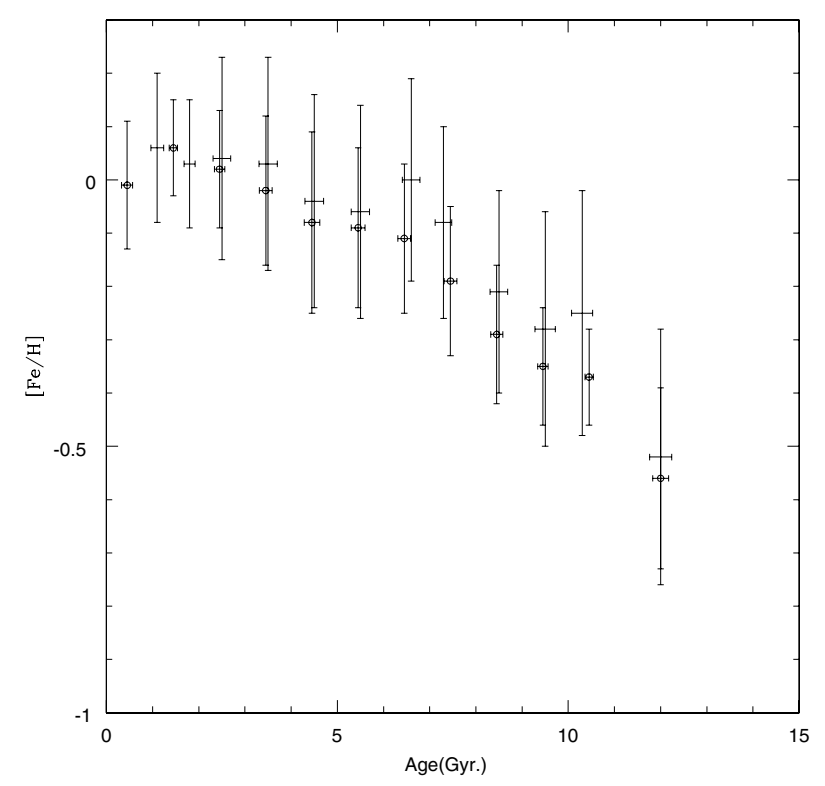

Fig. 2. Iron metallicity in the solar neighbourhood as a function of age. Observational comparison points with error bars are plotted from Meusinger et al. (1991, crosses) and Twarog (1986, circles plus signs).

a binning interval of $0.1 \mathrm{dex}$ for $[\mathrm{Fe} / \mathrm{H}]$ in this work. If we use a bin of 0.2 dex following VanDalfsen \& Harris (2004) although the two main peaks in the metal rich part of the $[\mathrm{Fe} / \mathrm{H}]$ diagram are not readily distinguishable, they do appear when the metallicity scale used is linear (see Figs. 1 and 2 of VanDalfsen \& Harris 2004).

Our basic scenario here takes into account the existence of two distinct populations of stars now observed in the halo and in the thick disc. The first population formed during the initial collapse of the Galaxy and the second accumulated during the disc lifetime, fed by star formation in the thin disc and was puffed up in height above the plane by dynamical inputs due to interactive activity (mergers or SN explosions, see below). The model presented here is aimed at explaining the metalrich part of the MDF of the globular clusters. It takes as its starting point two observationally derived relations: that of the local SFR as a function of epoch as presented in the work of Rocha-Pinto et al. (2000), presented in Fig. 2, and of the local iron metallicity as a function of epoch compiled from studies by Meusinger et al. (1991), Ibukiyama \& Arimoto (2002), Feltzing et al. (2001) and Bensby et al. (2004), shown in Fig. 3. The working hypothesis takes stellar numbers proportional to the peaks in the SFR of the disc, each star with its corresponding metallicity. The model asumes that when there was a peak in the disc SFR the resulting collective energy inputs from supernovae and winds of stars at the high mass end of the IMF were capable of driving quite massive gas clouds away from the Galactic plane (see Ikeuchi 1988). These clouds gave rise to star formation and in particular to stellar cluster formation, which we will model numerically here. The peaks observed by Rocha-Pinto et al. (2000) in the SFR of the thin disc is well explained by quasi-periodic fall of HVCs to the Galactic disc (see Casuso \& Beckman 1997; 2000, 2001, 2004). Both support against gravity and maintenance of observed motions appear

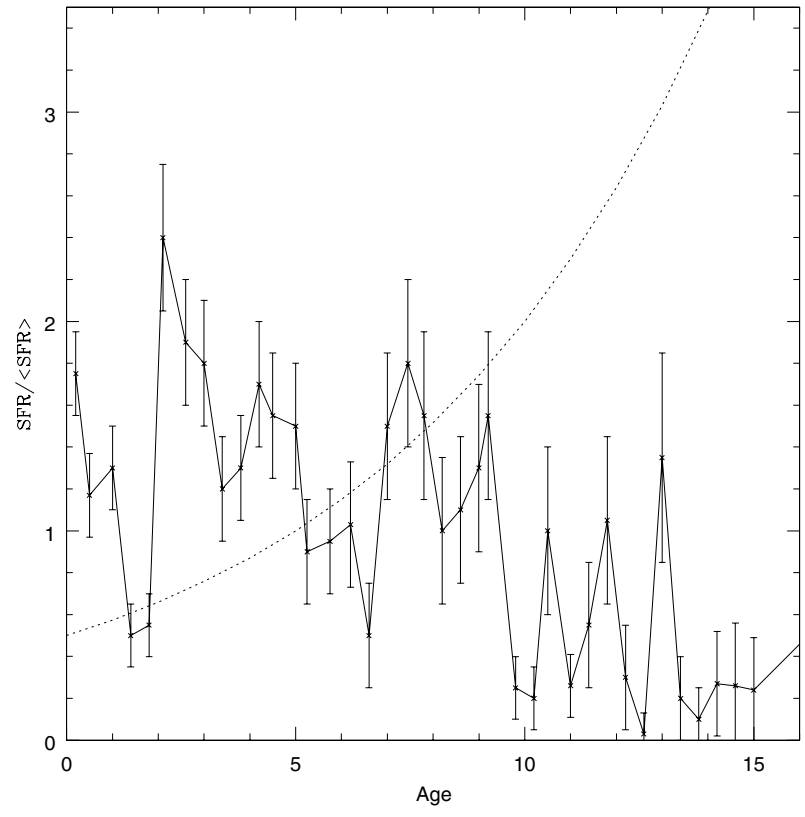

Fig. 3. History of the Galactic SFR. Data are in units of past average star formation, with error bars from Rocha-Pinto et al. (2000). The dashed line is the prediction of closed-box (no infall) model of star formation in the Galactic disc.

to depend on continued driving of the turbulence (Mac Low $\&$ Klessen 2004). A comprehensive parameter here is the ratio between the dissipation rate for isothermal, supersonic turbulence: $\frac{\rho_{\mathrm{ISM}} V_{\text {rms }}^{3}}{L_{\mathrm{d}}}$ where $L_{\mathrm{d}}$ is the driving scale, and the energy supplied by the shock front due to the HVC encounter with the ISM: $\frac{\rho_{\mathrm{HVC}} V_{\mathrm{HVC}}^{3}}{D}$ where $D$ is the length scale of the shock front. If this ratio is less than 1 it implies that the HVC collision breaks the cloud equilibrium and the gas cloud collapse to form stars. Taking tipical values for the parameters one obtain a range of values for the ratio between $10^{-3}$ for the HI gas clouds and 3 for the HII gas clouds, then implying that the HVCs collisions can account for well the main peaks observed for the SFR in the thin disc.

If we define $N\left([\mathrm{Fe} / \mathrm{H}]_{i}\right)$ as the number of stars which form in the thick disc from material expelled during one of the epochs of peak SFR in the disc, at which time the iron abundance of the star forming gas is $i$, then we have:

$N\left([\mathrm{Fe} / \mathrm{H}]_{i}\right)=\gamma \int_{0}^{T} \int_{T}^{t} n\left(\tau,[\mathrm{Fe} / \mathrm{H}]_{i}\right) \mathrm{d} \tau \mathrm{d} t$

where $T$ is the total age of the Galactic disc and $n\left(\tau,[\mathrm{Fe} / \mathrm{H}]_{i}\right)$ is the number of stars in each peak of the observed SFR in the disc itself, which we will take from the observations of Rocha-Pinto et al. (2000), and $\gamma$ is a normalization constant. The model results of this first step are presented in detail in Fig. 4, together with the observational data. The two main peaks in the higher metallicity lobe of the MDF for the globular clusters are reproduced in this model, but the direct association of peaks in this distribution with peaks in the SFR predict a third peak at near solar metallicity which is not observed at a statistically significant level (see Fig. 4).

We removed the third peak using a straightforward model in which stars are aggregated gravitationally during the path 


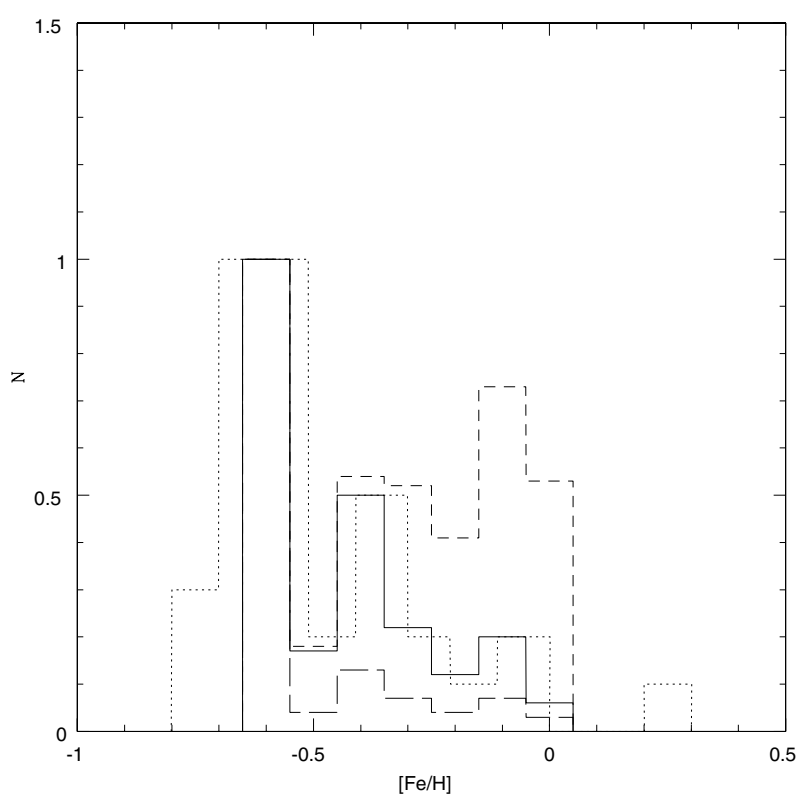

Fig. 4. Metallicity distribution function of metal-rich Galactic globular clusters. Data from Harris (1999, short dashed line). Model prediction with production of GCs inside the thin disc and then expulsed by SN explosions to the thick-disc and halo or GCs formed in the thick-disc inside a gas GMC expulsed from the thin-disc, in both cases following the SFR vs. age observed by Rocha-Pinto et al. (2000, dashed line). The same as previous but with a SFR vs. age modelled without infall of gas to the disc, and so increasing with age against the observations of Rocha-Pinto et al. (2000, long dashed line). The prediction of our model with production of open clusters and individual stars in the thin disc following the observed SFR of Rocha-Pinto et al. (2000), expulsed by SN to the thick disc and halo, and forming GCs by gravitational agregation during the sweeping path of some open clusters through the thin-disc over $\sim 5$ Gyr (full line).

of a cluster through the thin disc to the thick disc in a characteristic time of $\sim 5 \mathrm{Gyr}$, which is commensurate with the time between the SFR peaks observed by Rocha-Pinto et al. (2000), shown in Fig. 3. We assume a homogeneous distribution for individual stars, and also for the nuclei of gravitationaol aggregation, which are the gravitationally bound star groups. We also make the simplifying assumption that globular clusters are form from open clusters by more or less rapid aggregation of stars. The underlying mechanism we use is that of Mac Low \& Klessen (2004), whereby multiple SNe produce major shockwaves which accelerate and compress gas in giant molecular clouds. There are two types of dynamical effects relevant to the formation of the star clusters we are considering. One is the net impulse given to a GMC as a whole, and the other is the breaking of the equilibrium between self-gravity and driven supersonic turbulence which leads to collapse and star formation. If a star cluster encounters a GMC before it has aggregated a stellar mass of $10^{4} M_{\odot}$ to $10^{5} M_{\odot}$ it will disrupt and not go on to form a more massive cluster. So we can compute a probability for disruption which is proportional to the density of GMC's in the Galactic disc. Taking a constant volume to observe the formation of the Galactic disc assumed as a fall of gaseous giant HVCs from the intergalactic medium (IGM):

$$
\frac{\mathrm{d} M_{\mathrm{disc}}}{\mathrm{d} t} \simeq\left(V_{\mathrm{HVC}}+\int_{t_{r}}^{t_{D}} \frac{G M_{\mathrm{disc}}}{r^{2}} \mathrm{~d} t\right) \cdot \rho_{\mathrm{HVC}} \cdot S_{\mathrm{HVC}}
$$

where $V_{\mathrm{HVC}}$ is the velocity of an HVC in the IGM, and $t_{r}$ and $t_{D}$ are the time when a HVC is at a distance $r$ (normal to the disc plane) from the disc plane and the time when the HVC penetrates the thin disc tidal height, respectively. And due to the HVC density $\rho_{\mathrm{HVC}}$, HVC surface $S_{\mathrm{HVC}}$ and local disc mass $M_{\text {disc }}$ can be taken approximately constant at first order, one has $M_{\text {disc }} \propto\left(M_{0}+\mathrm{e}^{t / T_{0}}\right)$, where $M_{0}$ and $T_{0}$ are the initial mass of the disc and the characteristic time of the process. Then, we have a similar expresion for the density $\rho_{\text {disc }} \propto\left(\rho_{0}+\mathrm{e}^{t / T_{0}}\right)$. And so, we have for the probability of disruption for open clusters before GCs are formed, a proportionality to the same time exponential with the same characteristic time, and inverting we have that the probability of formation of globular clusters is proportional to the same time exponential but changing time by age. The value of the key constant $T_{0}$ is determined by the time interval between conditions favourable to the peaks in the SFR, which can be taken from the observed separation present in the data of Rocha-Pinto et al. (2000) and we will adopt a working value of $5 \mathrm{Gyr}$ for this parameter (see Fig. 3).

Then the number of metal-rich globular clusters $N_{\mathrm{GCs}}$ will vary with age, $t$ as

$\frac{\mathrm{d} N_{\mathrm{GCs}}}{\mathrm{d} t} \propto N_{\mathrm{GCs}}$

and this equation has the solution:

$N_{\mathrm{GCs}}\left([\mathrm{Fe} / \mathrm{H}]_{i}\right)=N\left([\mathrm{Fe} / \mathrm{H}]_{i}\right) \mathrm{e}^{\frac{t\left(\left[\mathrm{Fe} / \mathrm{H}_{j}\right)\right.}{T_{0}}}$

where $t\left([\mathrm{Fe} / \mathrm{H}]_{i}\right)$ is the age at each relevant value of the iron metallicity in the disc, which we have taken from the observational compilation of Meusinger et al. (1991).

In order to find a quantitative solution to the problem of nuclei of aggregation for the thick disc globular clusters we propose that they were formed during the events which expelled the GMCs from close to the Galactic plane via multiple SN explosions. During the epochs when the star formation rate was at its highest, represented by the peaks in the observed function of Rocha-Pinto et al. (2000) clusters are formed inside the GMCs which are expelled omnidirectionally. A small fraction of these clusters will be able to accumulate further stars and remain stable. Those which accompany gas clouds heights above the plane characteristic of the thick disc, and serve as cluster formation nuclei. A group of a few hundred stars can initiate in this way a cluster of $10^{5}$ to $10^{6}$ stars. Those clusters which stay very close to the plane have an enhanced probability of suffering many gravitational encounters with the massive and ubiquitous GMC's and such clusters are disrupted on timescales of less than $10^{8}$ years (see e.g. Binney \& Tremaine 1987). However those few clusters which rise out of the plane and can stimulate further star formation in out of plane gas clouds may accumulate enough stars this way to remain gravitationally bound. The fact that there are of order $10^{5}$ open clusters 
in the Galaxy but only a couple of hundred globular clusters is consistent with a secnario in which gobular clusters can form around undisrupted open clusters or open cluster remnants, but only those massive enought, and which are in suitable gas cloud surroundings can survive, and these increase their densities as they incorporate new stars to form a globular cluster.

We can carry out a numerical demonstration to see whether the nucleus of stars in the open cluster can serve as a suitable accretion core. If we let the tidal radius of the open cluster take a value of $20 \mathrm{pc}$ and take a representative value for the density of stars in the Galactic disc as $0.05 \mathrm{pc}^{-3}$ we can assume a path through the Galaxy of order $10 \mathrm{kpc}$ to $15 \mathrm{kpc}$ in length during a time interval commensurate with the disc lifetime, for the cluster formed under the conditions needed (i.e. inside a major gas cloud expelled from the plane during a period of maximum SFR), the number of stars accreted would be of order $10^{5}$ to $10^{6}$. It is not difficult to show that under the conditions relevant to this scenario the initial cluster is unlikely to have been disrupted by encounters with GMCs. Using the formalism of Binney \& Tremaine (1987) the time $t_{\mathrm{d}}$ for the disruption of a cluster is given by $t_{\mathrm{d}} \simeq 5.7 \times 10^{8}\left(\frac{M_{\text {cluster }}}{250 M_{\odot}}\right) \cdot\left(\frac{1 \mathrm{pc}}{r_{\text {cluster }}}\right)^{3} \mathrm{yr}$, and $\left(\frac{M}{r^{3}}\right)_{\mathrm{GC}} \simeq 80 \times\left(\frac{M}{r^{3}}\right)_{\mathrm{OC}}$, where the subscripts GC and OC mean globular cluster and open cluster respectively. This formula leads to a typical disruption time for an open cluster of order $5.7 \times 10^{8} \mathrm{yr}$, while for a globular cluster this time is of order $5 \times 10^{10} \mathrm{yr}$. (This is a time similar to that obtained by Dinescu et al. (1999) from a model in which the main disruption mechanism is two-body relaxation destruction rates). Our results are consistent with the relative general stability of the globular cluster population.

We can make an order of magnitude estimate for the amount of gas expelled by major SF episodes in the Galactic thin disc (within $350 \mathrm{pc}$ from the plane of symmetry) into the thick disc region (between $350 \mathrm{pc}$ and $1 \mathrm{kpc}$ ). Assuming a SN rate in the Galactic disc of $1 \mathrm{SN}$ per $50 \mathrm{yr}$ (Mac Low $\&$ Klessen 2004), the number of SN produced during a major peak of the SFR lasting $1.5 \mathrm{Gyr}$ will be $\frac{1.5 \times 10^{9}}{50}$ i.e. $3 \times 10^{7}$. We have to make assumptions about how much mass this can raise into the thick disc, but a plausible order of magnitude estimate can be made by simply assigning $1 M_{\odot}$ of material per SN expelled into the thick disc, which then gives us $3 \times 10^{7} M_{\odot}$ of material, a value which equals the total mass of observed metal rich GC's, i.e., $\sim \frac{1}{4} \times 150 \times 10^{6} \sim 3 \times 10^{7} M_{\odot}$. A slightly more refined estimate can be obtained by taking a mean SN remnant radius of $10 \mathrm{pc}$ and a mean gas density within an $\mathrm{SN}$ remnant of $0.001 M_{\odot}$ per cubic pc (Ikeuchi 1988) which gives a total gas mass for expulsion of $\sim 3 \times 10^{8} M_{\odot}$. However we must reduce this by a factor $\geq 3$ since we need the gas to be driven in the $z$ direction so we obtain an upper limit estimate this way of $\sim 10^{8} M_{\odot}$ which is consistent with the stellar mass in GC's as just shown.

Now we give an outline scenario of how an open cluster with $\sim 100$ stars can go on to accumulate as many as $10^{6}$ stars to form a globular cluster. We first note that of the $\geq 10^{5}$ open clusters which form during an episode of enhanced star formation in the disc, only a very small fraction survive tidal disruption. We consider one of these survivors which begins as a GMC accelerated by many SN explosions in the optimal direction, i.e., moving at a small angle to the disc plane, an angle which is optimal for the accumulation process. Although clusters which move away from the plane will tend to suffer less purely tidal disruption, they are subject to disruption by disc shocking if their orbits lead them to pass across the plane. However we consider the selected cluster to survive long enough to be present while further stars are formed around it either from its own GMC which has been pushed with it by the $\mathrm{SN}$ explosions due to some of its more massive initial members, or from other GMC's as it moves through them. Its capture radius $r_{\mathrm{S}}$ will increase with time as its mass increases, according to the relation $r_{\mathrm{S}} \simeq \frac{2 G M(t)}{\sigma^{2}}$ where $M(t)$ is the mass of the cluster at time $t$, and $\sigma$ is the velocity dispersion of stars in the cluster. If one assumes that the density of the cluster $\left(\rho_{\mathrm{S}}\right)$ remains approximately constant as it accretes stars, we can use a simple dynamical model to get an approximate expression for the variation of the cluster mass with time. The general expression will be:

$$
\delta M(t) \simeq \pi r_{\mathrm{S}}^{2} \frac{\mathrm{d} r}{\mathrm{~d} t} \rho_{\mathrm{S}} \mathrm{d} t
$$

and if we now incorporate our expression for $r_{\mathrm{S}}$ as a function of $M(t)$ and also assume a moving cluster slowed by dynamical friction so that $\frac{\mathrm{d} r}{\mathrm{~d} t} \simeq V_{0} \exp \left(-\frac{t}{t_{\mathrm{d}}}\right)$ where $V_{0}$ is the initial velocity and $t_{\mathrm{d}}$ the characteristic velocity decay time, we can integrate Eq. (5) to obtain

$$
M(t) \simeq \frac{\sigma^{4} t_{\mathrm{d}} \exp \left(\frac{t}{t_{\mathrm{d}}}\right)}{4 \pi G^{2} V_{0} \rho_{\mathrm{S}}} .
$$

We can then use a set of standard values for the fixed parameters: $t_{\mathrm{d}} \simeq 10^{8} \mathrm{yr}, \rho_{\mathrm{S}} \simeq 0.05 \mathrm{pc}^{-3}, V_{0} \simeq 2000 \mathrm{~km} \mathrm{~s}^{-1}$ (Ikeuchi 1988), and $\sigma \simeq 1-10 \mathrm{~km} \mathrm{~s}^{-1}$, and calculate the time needed to form a globular cluster, containing say $10^{6}$ stars, from an open cluster of say $10^{2}$ stars via this mechanism, i.e. following Eq. (6). The calculation gives a characteristic time of 5 Gyr. Now, we know that there are $\sim 10^{5}$ open clusters in the Galaxy. If we assume that these were formed during the last major peak in the SFR (see Fig. 3), we can calculate that the number of open clusters which can survive to form globulars in this time will be $\sim 10^{5} \exp \left(-\frac{5 \times 10^{9}}{5.7 \times 10^{8}}\right) \sim 20$. This result give interesting agreement with the observed total number of metal rich globular clusters, since there are two observed peaks in the SFR so that from this modelling exercise we would expect to find around 40 clusters. There is a current SFR peak but this will not give rise to GCs for several Gyr.

There is a marked observational trend relating kinematics with metallicity, in the sense that the more metal-poor population has a higher velocity dispersion and a lower rotational velocity than the metal-rich population (Minniti 1996). However it is important to note specifically that the metal-rich globular clusters within $4 \mathrm{kpc}$ from the Galactic center have their kinematics correlated with that of the HI in the bulge/bar (Minniti 1996; Cote 1999). As the GC's in general are old, (their ages are greater than 7 Gyr) all of those which formed in the disc but have moved to within $4 \mathrm{kpc}$ of the center are tidally dominated (gravitationally trapped) by the potential of the bulge and by the present epoch show bulge kinematics. It is known 
that Galactic dynamical friction brings clusters to the center of the system in a time of $\sim 10$ Gyr (Binney \& Tremaine 1987, p. 428) which is consistent with the scenario we present here. Our model can thus give a natural explanation for the kinematic observations of Minniti (1996) and Cote (1999). There is also evidence from external galaxies that metal rich GC's within a few kpc of the center of a galaxy show bulge/bar kinematics (Fobres et al. 2001). Uncertainties in the present three dimensional galactocentric positions for most of the GC's do not yet permit an unambiguous discrimination between their belonging to a rigidly rotating bar or to a bulge which may be an oblate isotropic rotator (Cote et al. 1999).

It is of considerable interest for the present scenario that Morrison et al. (2004) have recently found that there is a subsytem of GC's in M 31 which clearly show thin disc kinematics. These clusters are found across the entire disc of M 31 and form some $40 \%$ of the clusters projected against the disc, which certainly indicates a thin disc origin of this population of GC's.

\section{Comparison with observations}

In Fig. 4 we can see how the present model gives an excellent fit to the main peak of the MDF of Galactic globular clusters. The observed peak for the thick disc component of this MDF falls close to $[\mathrm{Fe} / \mathrm{H}] \simeq-0.6$ which is well predicted here. The detailed model also predicts the subsidiary peak observed at $[\mathrm{Fe} / \mathrm{H}] \simeq-0.4$. The good correlation of our model with the observed MDF of the globular clusters is based on the observational peaks of the MDF of disc stars by Rocha-Pinto et al. (2000) which occur at disc metallicities which correspond rather well (Meusinger et al. 1991; Twarog 1986) to those required for the globular clusters. Thus the present model requires either the interaction of dwarf galaxies with the Galactic disc or the accretion of HVC's to induce the increases in the SFR noted in the SF peaks, which lead to the formation of open clusters and subsequently of globular clusters. Although we prefer the hypothesis of the HVC's, since there is considerable evidence from chemical evolution that not only has star formation been triggered but that low metallicity gas has been continually incorporated into the Galactic disc, (Casuso \& Beckman $1997,2000,2001,2004)$ we would not wish to rule out minor galaxy interactions as orginators of at least some of the SF peaks and hence of the peaks in the GC metallicity distribution. In Fig. 5 we show the predictions of a model with a minor galaxy merger, which does give a fair account of the main peak in the MDF of the thick disc GC's.

Although there are few observations the fact that these indicate values of $[\mathrm{Mg} / \mathrm{Fe}]$ close to 0.3 for GC's (see below) together with the clear observational tendency in the disc population for $[\mathrm{Mg} / \mathrm{Fe}]$ to increase with decreasing metallicity (Abia \& Mashonkina 2004) or with increasing age (Casuso et al. 1996) give a clear indication that the globular clusters we are pinpointing in this article have ages greater than $7 \mathrm{Gyr}$ and could therefore well have been formed in the thick disc from gas expelled in SN explosions in the major star forming episodes in the thin disc. Yong et al. (2003) reported observed abundances in 20 bright giants in the globular cluster NGC 6752. The dispersions for $[\mathrm{O} / \mathrm{Fe}],[\mathrm{Na} / \mathrm{Fe}]$ and $[\mathrm{Al} / \mathrm{Fe}]$ are

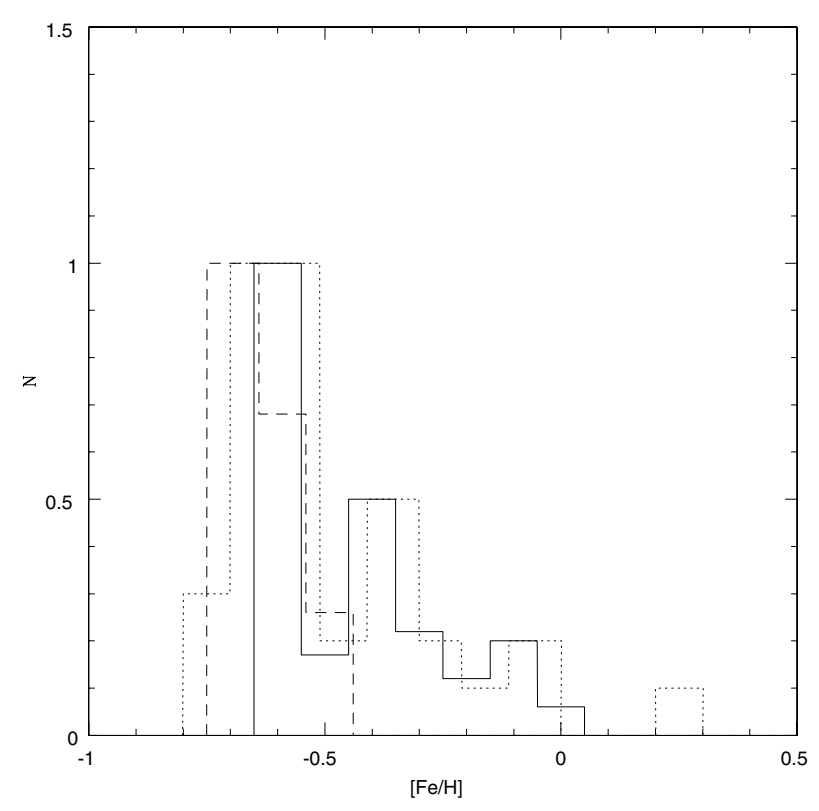

Fig. 5. Metallicity distribution function of metal-rich Galactic globular clusters. Data from Harris (1999, short dashed line). Model prediction based on the formation of disc globular clusters in early dissipative minor merging (Bekki \& Chiba 2002). The prediction of our model with production of open clusters and individual stars in the thin disc following the observed SFR of Rocha-Pinto et al. (2000), expulsed by SN to the thick-disc and halo, and forming GCs by gravitational agregation during the sweeping path of some open clusters through the thin-disc over $\sim 5$ Gyr (full line).

high, but for $[\mathrm{Mg} / \mathrm{Fe}]$ all the values lie between +0.3 and +0.5 , so that it does appear that this is the most reliable index for studying the $[\mathrm{alpha} / \mathrm{Fe}]$ ratio in the GC's.

\section{Summary}

In the decades prior to the 1990's it was generally believed that very different mechanisms were needed to explain the formation of open clusters and globular clusters. The current consensus is that all clusters form in a basically similar way, but that globular clusters form preferentially in regions of high ISM pressure and high SFR (Elmegreen \& Efremov 1997; Ashman \& Zepf 2001). It would not be correct to claim uniqueness for our model reproduction of the observed MDF of the globular clusters in the Milky Way. A key restriction here is the dispersion in the age-metallicity relation, which could displace the peaks in both the observations and the model by as much as 0.2 dex in [Fe/H] (Ibukiyama \& Arimoto 2002; Feltzing et al. 2001; Bensby et al. 2004). While we do not doubt that the process of galaxy mergers gives rise to star formation, and could specifically lead to the formation of globular clusters, chemical evolution models for the Milky Way do seem to point to the existence of long term low metallicity gas inflow, and the embodiment of this inflow in the HVC's leads naturally to an alternative mechanism. Here we propose that during episodes of the most enhanced star formation in the Galaxy a small but significant fraction of the open clusters are effectively either expelled to thick disc altitudes or are formed at these altitudes, and are able to survive there undisrupted for periods 
long enough for them to nucleate globular clusters by gravitational stellar accretion. This mechanism gives us not only a qualitative picture of how the more metallic globular clusters were formed, but allows us to explain their observed MDF in considerable quantitative detail.

Acknowledgements. We are happy to thank the anonymous referee for very helpful comments and suggestions. This research was supported by grant AYA 2004-08251-C02-01 of the Spanish Ministry of Science and Education.

\section{References}

Abia, C., \& Mashonkina, L. 2004, MNRAS, 350, 1127

Armandroff, T. E. 1989, AJ, 97, 375

Ashman, K. M., \& Zepf, S. E. 1992, ApJ, 384, 50

Ashman, K. M., Bird, C. M., \& Zepf, S. E. 1994, AJ, 108, 2348

Ashman, K. M., \& Zepf, S. E. 2001, AJ, 122, 1888

Bekki, K., \& Couch, W. J. 2001, ApJ, 557, L19

Bekki, K., \& Chiba, M. 2002, ApJ, 566, 245

Bekki, K., Forbes, D. A., Beasley, M. A., \& Couch, W. J. 2002, MNRAS, 335, 1176

Bekki, K., Couch, W. J., Beasley, M. A., et al. 2004, ApJ, 610, L93

Bensby, T., Feltzing, S., \& Lundstrom, I. 2004, A\&A, 421, 969

Binney, J., \& Tremaine, S. 1987, in Galactic Dynamics (USA: Princeton University Press), 26

Carney, B. W., Latham, D. W., \& Laird, J. B. 1990, AJ, 99, 572

Casuso, E., Vazdekis, A., Peletier, R. F., \& Beckman, J. E. 1996, ApJ, 458,533

Casuso, E., \& Beckman, J. E. 1997, ApJ, 475, 155

Casuso, E., \& Beckman, J. E. 2000, PASP, 112, 942

Casuso, E., \& Beckman, J. E. 2001, ApJ, 557, 681
Casuso, E., \& Beckman, J. E. 2004, A\&A, 419, 181

Cote, P. 1999, AJ, 118, 406

Dinescu, D. I., Girard, T. M., \& van Altena, W. F. 1999, AJ, 117, 1792

Elmegreen, B. G., \& Efremov, Y. N. 1997, ApJ, 480, 235

Feltzing, S., Holmberg, J., \& Hurley, J. R. 2001, A\&A, 377, 911

Harris, W. E. 1976, AJ, 81, 1095

Harris, W. E. 1996, AJ, 112, 1487

Harris, W. E. 1999, in Globular Clusters (CUP)

Heasley, J. N., Janes, K. A., Zinn, R., et al. 2000, AJ, 120, 879

Ibukiyama, A., \& Arimoto, N. 2002, A\&A, 394, 927

Ikeuchi, S. 1988, Fund. Cosmic Phys., 12, 255

Jehin, E., Magain, P., Neuforge, C., Noels, A., \& Thoul, A. A. 1998, A\&A, 330, L33

Kennicutt, R. C., Jr. 1998, ARA\&A, 36, 189

Kinman, T. D. 1959, MNRAS, 119, 538

Li, Y., Maclow, M., \& Klessen, R. S. 2004, ApJ, 614, L29

Mac Low, M. M., \& Klessen, R. S. 2004, Rev. Mod. Phys., 76, 125

Majewski, S. R. 1994, ApJ, 431, L17

Meusinger, H., Reimann, H., \& Stecklum, B. 1991, A\&A, 245, 51

Minniti, D. 1996, ApJ, 459, 175

Morrison, H. L., Harding, P., Perrett, K., \& Hurley-Keller, D. 2004, ApJ, 603, 87

Noguchi, M., \& Ishibashi, S. 1986, MNRAS, 219, 305

Robin, A. C., Haywood, M., Creze, M., Ojha, D. K., \& Bienayme, O. 1996, A\&A, 305, 125

Rocha-Pinto, H. J., Scalo, J., Maciel, W. J., \& Flynn, C. 2000, A\&A, 358,869

Twarog, B. A. 1986, ApJ, 242, 242

VanDalfsen, M. L., \& Harris, W. E. 2004, AJ, 127, 368

Vandenberg, D. A. 2000, ApJSS, 129, 315

Yong, D., Grundahl, F., Lambert, D. L., Nissen, P. E., \& Shetrone, M. D. 2003, A\&A, 402, 985

Zinn, R. 1985, ApJ, 293, 424 